Check for updates

Cite this: Nanoscale Adv., 2019, 1, 3597

\title{
High-throughput chemical and chemoenzymatic approaches to saccharide-coated magnetic nanoparticles for MRI $\dagger$
}

\author{
Thomas W. Fallows, (D) ab Andrew J. McGrath, (D) ${ }^{c d}$ Joana Silva, (D) ab \\ Simon G. McAdams, ${ }^{\text {ae }}$ Andrea Marchesi, (iD ab Floriana Tuna, (iD af Sabine L. Flitsch, (iD ab \\ Richard D. Tilley (D) ${ }^{\text {cdg }}$ and Simon J. Webb (D) *ab
}

\begin{abstract}
There is a need for biofunctionalised magnetic nanoparticles for many biomedical applications, including MRI contrast agents that have a range of surface properties and functional groups. A library of eleven adducts, each formed by condensing a reducing sugar with a catechol hydrazide, for nanoparticle functionalisation has been created using a high-throughput chemical synthesis methodology. The enzymatic transformation of an $\mathrm{N}$-acetylglucosamine (GlcNAc) adduct into an $\mathrm{N}$-acetyllactosamine adduct by $\beta-1,4$-galactosyltransferase illustrates how chemoenzymatic methods could provide adducts bearing complex and expensive glycans. Superparamagnetic iron oxide nanoparticles ( $8 \mathrm{~nm}$ diameter, characterised by TEM, DLS and SQUID) were coated with these adducts and the magnetic resonance imaging (MRI) properties of GlcNAc-labelled nanoparticles were determined. This straightforward approach can produce a range of MRI contrast agents with a variety of biofunctionalised surfaces.
\end{abstract}

Received 14th June 2019

Accepted 29th July 2019

DOI: $10.1039 / c 9 n a 00376 b$

rsc.li/nanoscale-advances

surfaces could be tailored post synthesis using different coating

\section{Introduction}

Cell targeting magnetic nanoparticles (MNPs) with the ability to bind to specific cell types have many current and potential applications, including in magnetic resonance imaging (MRI), ${ }^{1}$ magnetic hyperthermia, ${ }^{2,3}$ and magnetic separations. ${ }^{4,5}$ A popular current approach towards cell targeting is use of antibody-coated nanoparticles, but several issues exist with this approach, particularly cost. ${ }^{6}$ Cell-cell recognition processes suggest an alternative approach, as cell adhesion is often mediated through recognition of an ensemble of cell surface glycans (oligosaccharides). ${ }^{7}$ This produces highly specific but tuneable recognition of different cell types, which has made the surface glycosylation of nanoparticles of particular interest. ${ }^{8}$ If libraries of saccharideterminated ligands for MNPs were available, then nanoparticle

${ }^{a}$ School of Chemistry, University of Manchester, Oxford Road, Manchester M13 9PL, UK. E-mail: S.Webb@manchester.ac.uk; Tel: +44 (0)161 3064524

${ }^{b}$ Manchester Institute of Biotechnology, University of Manchester, 131 Princess St, Manchester M1 7DN, UK

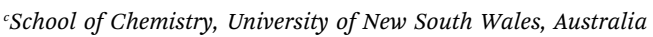

${ }^{d}$ Australian Centre for NanoMedicine, University of New South Wales, Australia ${ }^{e}$ School of Materials, University of Manchester, Oxford Road, Manchester, UK ${ }^{f}$ Photon Science Institute, University of Manchester, Oxford Road, Manchester M13 9PL, UK

${ }^{g}$ Electron Microscope Unit, Mark Wainwright Analytical Centre, University of New South Wales, Australia

$\dagger$ Electronic supplementary information (ESI) available: Synthetic procedures and copies of NMR spectra. Additional MNP characterisation data. See DOI: 10.1039/c9na00376b ligands that are mixed in situ, allowing optimised binding to the suite of cell surface lectins on a targeted cell line.

One interesting application of cell-targeting saccharidecoated MNPs would be in vivo labelling agents for MRI. MRI is a well-established diagnostic tool for imaging tissues within the body ${ }^{9}$ that circumvents the use of contrast agents with short half-lives (as in positron emission tomography) ${ }^{10}$ and offering better resolution at greater depths than is achievable by ultrasound. ${ }^{11}$ MRI contrast agents increase differences in the $T_{1}$ (spin-lattice) and $T_{2}$ (spin-spin) relaxivity of water protons ${ }^{1}$ in different tissues, allowing better imaging of internal organs. $\mathrm{Gd}^{3+}$ complexes are currently the most commonly used MRI contrast agents and are primarily useful for $T_{1}$-weighted images, but $\mathrm{Gd}^{3+}$ leeching in the body has been suggested as a cause of nephrogenic systemic fibrosis. ${ }^{12}$ Superparamagnetic iron oxide nanoparticles (SPIONs) are an alternative that act as $T_{2}$ contrast agents. ${ }^{13}$ SPIONs can be surface functionalised (for example with catechol derivatives) to improve stability, to increase circulation time, to add therapeutic agents, or to introduce targeting groups. ${ }^{14}$ Beyond labelling tissues for MRI, biofunctionalised MNPs might be useful for magnetic biosensing, ${ }^{15}$ where an in situ binding process gives a change in magnetic signal. For example, it has been shown that using lectins to aggregate saccharide-coated nanoparticles leads to a measureable decrease in the $T_{2}$ value. ${ }^{16}$ It may be possible to image similar aggregation processes in vivo, perhaps allowing the real-time tracking of biochemical processes on MNP 


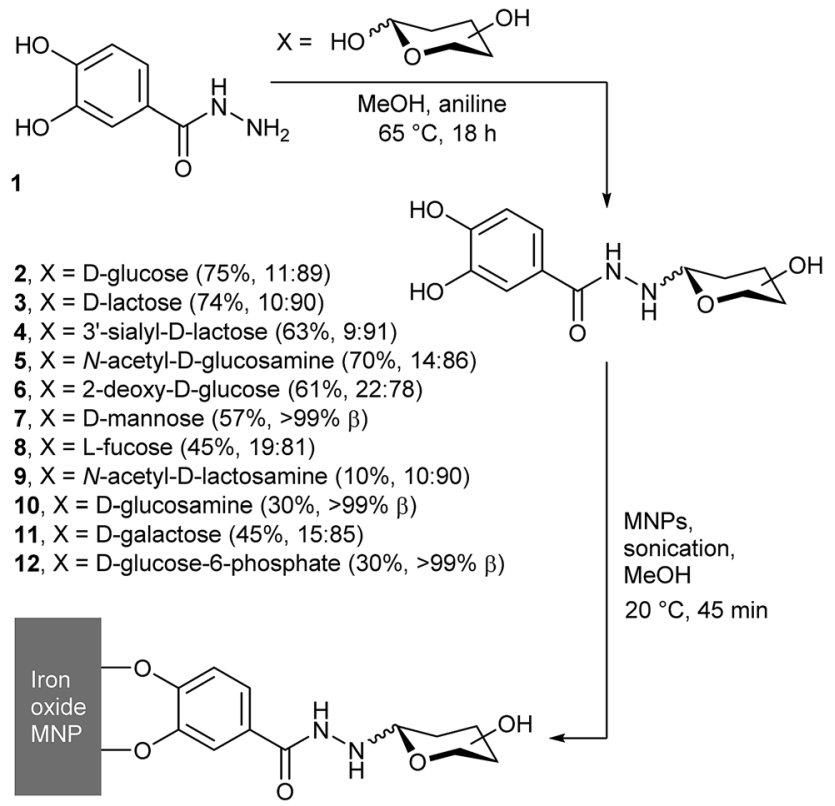

Fig. 1 General scheme showing condensation of 3,4-dihydroxybenzhydrazide with reducing sugars, to give conjugates $2-12$. In brackets after each compound is the yield and the $\alpha: \beta$ anomeric ratio.

surfaces or MNP agglomeration on targeted cancerous cells in the early stages of tumour development.

We have recently shown that condensing catechol hydrazide 1 with four different reducing sugars gave the corresponding saccharide-catechol conjugates ( 2 to 5 , Fig. 1$).{ }^{17}$ Purification of the crude mixtures by high-performance liquid chromatography (HPLC) gave the four adducts in a high-throughput process, with costs commensurate with that of the starting saccharide. These adducts were coated onto the surface of MNPs, to give saccharidecoated MNPs designed to exploit the affinity of cell-surface lectins for specific saccharide motifs. These coatings were found to be quite stable; hydrolysis of the hydrazone link in the resorcinol analogues is slow at pH 7.4 (ca. 25\% after 24 days) and desorption of the catechol group from a MNP surface is even slower $\left(t_{1 / 2}\right.$ for dissociation $>8$ weeks). ${ }^{17}$ Both cell-surface and lectin recognition of these coated MNPs has been shown, ${ }^{17}$ with cell targeting by glucose (Glc) and $N$-acetylglucosamine (GlcNAc) labelled MNPs used to impart magnetophoretic behaviour on 3T3 fibroblasts. ${ }^{18}$

To grow these four published examples into a library, we have explored the reactivity of another nine reducing saccharides with catechol hydrazide 1 . We then explored the potential of chemoenzymatic synthesis to expand this library and include more complex and expensive saccharides. SPIONs were coated with these saccharide-catechol conjugates and the potential of saccharide-coated SPIONs as MRI contrast agents was explored, which included the effect of adding a conjugate lectin.

\section{Results and discussion}

\section{Chemical synthesis of saccharide adducts 2-12}

We previously reported that the aniline-catalysed reaction of 3,4-dihydroxybenzhydrazide 1 with glucose, $\mathrm{N}$ - acetylglucosamine, lactose, and $3^{\prime}$-sialyllactose gives the corresponding hydrazones, which cyclise to form the pyranoside adducts. ${ }^{17}$ The same simple, high-throughput method (Fig. 1) was now applied with D-sugars 2-deoxyglucose, mannose, $\mathrm{N}$ acetyllactosamine (LacNAc), glucosamine, galactose, glucose-6phosphate, Lewis ${ }^{\mathrm{X}}$ trisaccharide and Lewis $^{\mathrm{X}}$ tetrasaccharide, as well as L-fucose.

In addition to aniline, other catalysts for hydrazone and oxime formation were tested, such as those assessed by Wendeler et $a l .{ }^{19}$ and Crisalli et al. ${ }^{20} p$-Phenylenediamine and anthranilic acid were chosen due to their combination of high reported catalytic activity and relatively low cost. Reactions between $\mathbf{1}$ and glucose in NMR tubes were carried out in $\mathrm{CD}_{3} \mathrm{OD}$ at $65{ }^{\circ} \mathrm{C}$ for each catalyst, as well as a control with no catalyst present. ${ }^{1} \mathrm{H}$ NMR spectra were measured at $0,1,2,4,6,8$ and $24 \mathrm{~h}$ and the yields were calculated by integrating the anomeric peaks of glucose and the glucose adduct 2 (see the ESI†). These studies showed that an overnight reaction gave the optimal balance between reaction time and yield, as rapid equilibration of the glucose anomers was followed by a steady increase in the fraction of adduct $\mathbf{2}$ formed over the $24 \mathrm{~h}$. This reached $20 \%$ for the uncatalysed reaction, but both $p$-phenylenediamine and anthranilic acid gave in situ conversions in excess of $80 \%$ (see the ESI $\dagger$ ). These conversions were better than that of aniline (55\%), but after reversed-phase HPLC purification an aniline catalyst was found to give the purest product; this catalyst was favoured thereafter.

Purification of the products 6-12 by reversed-phase HPLC separation was most effective if multiple aliquots $(0.5 \mathrm{~mL})$ were separated on a semi-preparative column; a single crude reaction mixture (ca. $60 \mathrm{mg}$ ) could usually be purified during one day. The collected fractions were then concentrated under reduced pressure to remove the organic solvent, before being lyophilised to give the products as white powders. The products were analysed by ${ }^{1} \mathrm{H}$ NMR spectroscopy, which showed no resonances from open chain hydrazone (typically found at $6.5-8 \mathrm{ppm})^{21}$ but resonances in the 3.7-4.7 ppm region from the anomeric protons of ring closed pyranosides. This region of the spectra showed that our HPLC conditions did not separate the $\alpha$ - and $\beta$ anomers of the adducts. However, integration of the resonances provided the $\alpha: \beta$ ratio (see the ESI $\dagger$ ) in the purified mixtures, which showed the $\beta$-anomer was favoured, from $78 \%$ for 6 to $>99 \%$ for 7,10 and 12 (Fig. 1).

Under these reaction conditions, the yields for the condensation of 1 with uncharged saccharides were fair overall (generally 40 to $70 \%$ ), except in the case of $N$-acetyllactosamine, which only gave a low yield of the adduct (yield $=(10 \pm 2) \%$ ). More complex adducts, such as those with Lewis ${ }^{\mathrm{x}}$ trisaccharide and Lewis ${ }^{\mathrm{X}}$ tetrasaccharide, could not be obtained in useful quantities due to low conversions combined with the high cost of the starting sugars. The yields in these cases were no greater than $13 \%$ and $35 \%$ respectively, although the very small amounts obtained ( 0.7 and $1.2 \mathrm{mg}$ respectively) precluded full characterisation. Analysis of the by-products of these reactions also provided evidence of fragmentation of the oligosaccharides under the reaction conditions. In particular, the fucose adduct 8 (identified by ${ }^{1} \mathrm{H}$ NMR spectroscopy) was isolated from the crude mixture of Lewis ${ }^{\mathrm{X}}$ tetrasaccharide in $10 \%$ yield $(0.2 \mathrm{mg})$. 


\section{Enzymatic synthesis of saccharide adduct 9}

Given the low yield for LacNAc adduct 9, chemoenzymatic methods for synthesising this compound were explored. Glycosyltransferases give excellent regio- and stereochemical control, and several glycosyltransferases have been shown to act on unnatural substrates, including substrates immobilised on surfaces. ${ }^{22} \quad \beta-1,4$-Galactosyltransferase 1 ( $\beta 4$ Gal-T1) has been shown to be particularly versatile in this respect, for example adding galactose to $N$-acetylglucosamines displayed on gold surfaces, ${ }^{23}$ glass surfaces, ${ }^{24}$ and lipid bilayers ${ }^{25,26}$ to give LacNAc labelled surfaces. Should GlcNAc adduct 5 be a substrate for $\beta 4 \mathrm{Gal}-\mathrm{T} 1$, there is also the potential for this transformation to be performed in situ on the nanoparticle surface.

To assess if $\beta 4 \mathrm{Gal}-\mathrm{T} 1$ could transform GlcNAc adduct 5 into LacNAc adduct $\mathbf{9}$, conditions that had been employed previously on synthetic GlcNAc glycolipids were applied (Fig. 2a). ${ }^{25,26}$ The GlcNAc adduct was dissolved in MES buffer (1 mL) along with $\beta 4 \mathrm{Gal}-\mathrm{T} 1$ enzyme ( $14.75 \mu \mathrm{L}$ of a $0.54 \mathrm{mg} \mathrm{mL}^{-1}$ solution), uridine diphosphogalactose disodium salt (UDP-Gal, $11.25 \mathrm{mg}, 20$ $\mu \mathrm{mol})$ and $\mathrm{MnCl}_{2}$ (3 $\mu \mathrm{L}$ of a $1 \mathrm{M}$ solution in water). The mixture was incubated at $37^{\circ} \mathrm{C}$ overnight. After incubation, the reaction mixture was analysed by positive ion LCMS, which showed two ions with $\mathrm{m} / \mathrm{z}$ slightly lower than that expected for the LacNAc product; $[\mathbf{9}+\mathrm{H}-2 \mathrm{H}]^{+}$and $[\mathbf{9}+\mathrm{Na}-2 \mathrm{H}]^{+}$. The loss of two hydrogens was hypothesized to be due to $\mathrm{Mn}$ (II)-catalysed aerial oxidation of the catechol moiety in $\mathbf{5}$, as the solution turned light brown after $\mathrm{MnCl}_{2}$ addition but remained colourless in the absence of $\mathrm{MnCl}_{2}$. Mixing 1 with $\mathrm{MnCl}_{2}$ in $\mathrm{MES}$ buffer in air gave the same colour change, but degassing of the solution followed by purging with argon before $\mathrm{MnCl}_{2}$ addition prevented this oxidation.

Repetition of the enzymatic transformation using samples under an Ar atmosphere, followed by LCMS, revealed that ions corresponding to the LacNAc product, $[\mathbf{9}+\mathbf{H}]^{+}$and $[9+$ $\mathrm{Na}]^{+}$, were found in a mixed fraction that eluted at 12 minutes (Fig. 2b). Further attempts to purify this mixed fraction of 5 and 9 using other HPLC conditions still resulted in co-elution of 5 and 9. To determine the extent of the enzymatic transformation, the fraction containing $\mathbf{5}$ and $\mathbf{9}$ was analysed by ${ }^{1} \mathrm{H}$ NMR spectroscopy. The 3.7-4.7 ppm region contains resonances from the $\alpha$ and $\beta$ anomeric protons $\left(\mathrm{H}_{\mathrm{a}}(\alpha)\right.$ and $\mathrm{H}_{\mathrm{a}}(\beta)$ respectively) on the glucosyl moieties of both $\mathbf{5}$ and $\mathbf{9}$, as well as the exclusively $\beta$ anomeric proton $\left(\mathrm{H}_{\mathrm{b}}\right)$ on the galactosyl moiety of 9 . The integrations of the anomeric protons $\mathrm{H}_{\mathrm{a}}(\alpha), \mathrm{H}_{\mathrm{a}}(\beta)$ and $\mathrm{H}_{\mathrm{b}}$ in 5 and 9 were $0.16,0.92$ and 0.33 , respectively (Fig. 2c), which suggests that the conversion of 5 to 9 was about $30 \%$ after $24 \mathrm{~h}$. This value was significantly lower than the $40 \%$ conversion after $1 \mathrm{~h}$ reported for the conversion of $p$-nitrophenyl-GlcNAc to $p$-nitrophenylLacNAc using $\beta 4 \mathrm{Gal}-\mathrm{T} 1$ and UDP-Gal, and more similar to conversions obtained for synthetic GlcNAc-capped glycolipids (10-30\% after $6 \mathrm{~h}) .{ }^{25}$ The overall percentage conversion was superior to that obtained by the chemical synthesis of adduct $\mathbf{9}$, although pure $\mathbf{9}$ has not yet been obtained using our HPLC method. a)
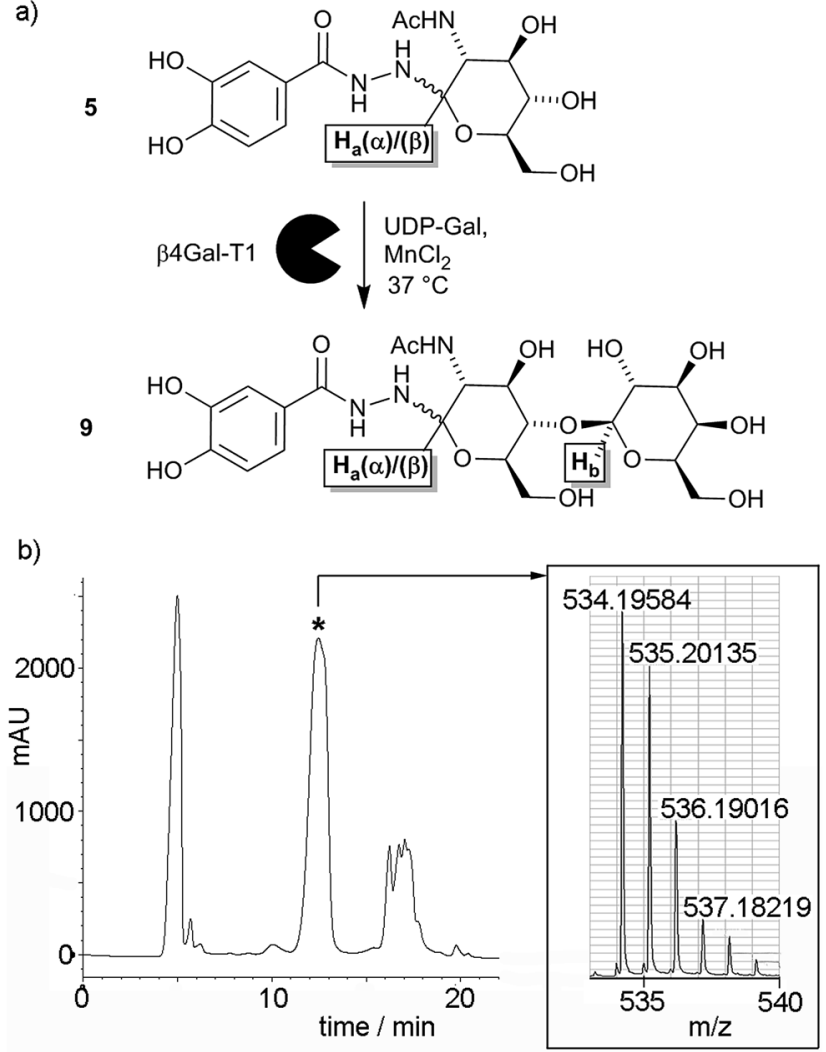

c)

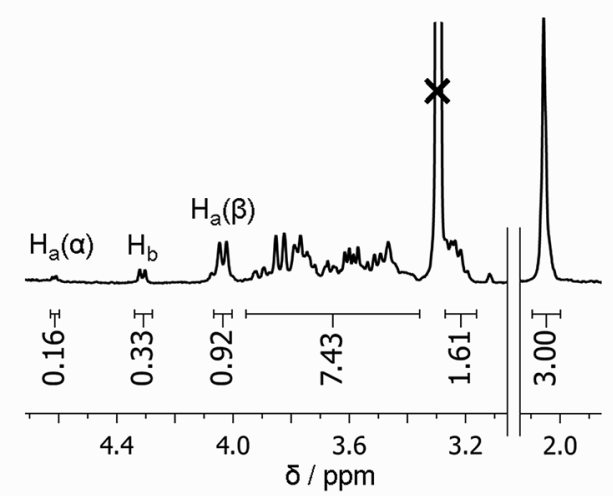

Fig. 2 (a) Enzymatic transformation of GlcNAc adduct 5 into LacNAc adduct 9. (b) HPLC trace for the enzymatic transformation of 5 into 9 , with the product containing fraction indicated (*). Inset: MS data indicating the enzymatic formation of 9. (c) Partial ${ }^{1} \mathrm{H}$ NMR spectrum for the enzymatically produced mixture of 5 and 9 , showing integrations of the anomeric proton resonances (as labelled in (a) above) relative to the integration of the methyl resonance $(3 \mathrm{H}$, shown right).

\section{Synthesis of saccharide-coated MNPs}

Both $_{\text {chemical }}{ }^{27}$ and enzymatic routes ${ }^{28}$ for iron oxide MNP synthesis exist. The simplest chemical route to iron oxide MNPs is the co-precipitation method, ${ }^{29}$ where $\mathrm{Fe}^{3+}$ and $\mathrm{Fe}^{2+}$ are mixed in a $2: 1$ ratio at basic $\mathrm{pH}$, and there are a number of published methods that give good size control. ${ }^{30}$ In the absence of oxygen, the iron oxide formed is magnetite $\left(\mathrm{Fe}_{3} \mathrm{O}_{4}\right)$, but this can be readily oxidised on the surface ${ }^{31}$ and in bulk to maghemite $(\gamma$ $\left.\mathrm{Fe}_{2} \mathrm{O}_{3}\right) \cdot{ }^{32}$ 
Iron oxide nanoparticles were synthesised by the coprecipitation method, and stored under $\mathrm{N}_{2}$ to prevent oxidation. To coat the MNPs, the appropriate saccharide-catechol adduct $(10 \mathrm{mg})$ was added to MNPs $(10 \mathrm{mg})$ in methanol $(5 \mathrm{~mL})$ and the suspension was probe sonicated for $45 \mathrm{~min}$. The coated MNPs were then sedimented by centrifugation and the supernatant was removed with the aid of a permanent magnet, which held and avoided disturbance of the MNP pellet. The nanoparticles were washed with methanol to remove any unbound adduct before re-suspension by bath sonication. The process of sedimentation and washing was repeated twice with methanol and once with Milli-Q filtered water. The coated nanoparticles were finally suspended in Milli-Q filtered water and lyophilised for storage. ${ }^{17}$

\section{Characterisation of uncoated and coated MNPs}

The type of iron oxide in the MNPs can be inferred from the Xray powder diffraction (XRD) pattern. This information can be further supplemented with high-resolution transmission electron microscopy (TEM), which not only provides a measure of particle diameter but may also show the crystalline structure of the iron oxide. XRD patterns obtained for MNPs coated with 212 were very similar to each other and to that of the uncoated nanoparticles (see the ESI $\dagger$ ). For example, the XRD patterns of uncoated and GlcNAc-coated MNPs are similar (Fig. 3a) and comparable to both magnetite and maghemite. The XRD of maghemite, however, has weak peaks at $23.77^{\circ}(210)$ and $26.10^{\circ}$ $(211)^{33}$ and there are no clear peaks in this $20-30^{\circ}$ region in the XRD pattern of the MNPs, which may suggest these MNPs are magnetite. Nonetheless both magnetite and maghemite nanoparticles are known to exhibit superparamagnetism. ${ }^{34}$ Inductively Coupled Plasma Atomic Emission Spectroscopy (ICP-AES) was then used to estimate the extent of coating on the nanoparticle surface. Phosphorus can be quantified by ICP-AES, therefore MNPs coated with the (glucose-6-phosphate)-catechol adduct 12 were investigated. These coated MNPs had $1.64 \% \mathrm{w} / \mathrm{w}$ phosphorus, which shows 12 covers around $59 \%$ of the available surface area of each MNP (see the ESI $\dagger$ ); this value is similar to that found for another catechol-based conjugate coated on MNPs. ${ }^{35}$

Nanoparticle size is an important factor for determining both biological responses to particles and their magnetic behaviour; the formation of single magnetic domains will give superparamagnetic behaviour. TEM of the synthesised MNPs showed that most particles were smaller than $20 \mathrm{~nm}$ in diameter (Fig. 3b), with an average size of $8.3 \mathrm{~nm}$ (standard deviation $2.4 \mathrm{~nm}, n=100$, see $\mathrm{ESI} \dagger$ ), which is in a range typical for particles formed by this method..$^{29,36}$ Little difference was observed by TEM between coated and uncoated nanoparticles, and both appeared to agglomerate into small clusters around $200 \mathrm{~nm}$ in diameter. The GlcNAc-coated MNPs, however, showed a visible 'corona' around the nanoparticle clusters (Fig. 3c), something that is often suggestive of carbonaceous coatings. ${ }^{37}$ Interestingly, despite finding GlcNAc-coated MNPs bound to Quartz Crystal Microbalance with Dissipation monitoring (QCM-D) chips coated with the GlcNAc-recognising lectin

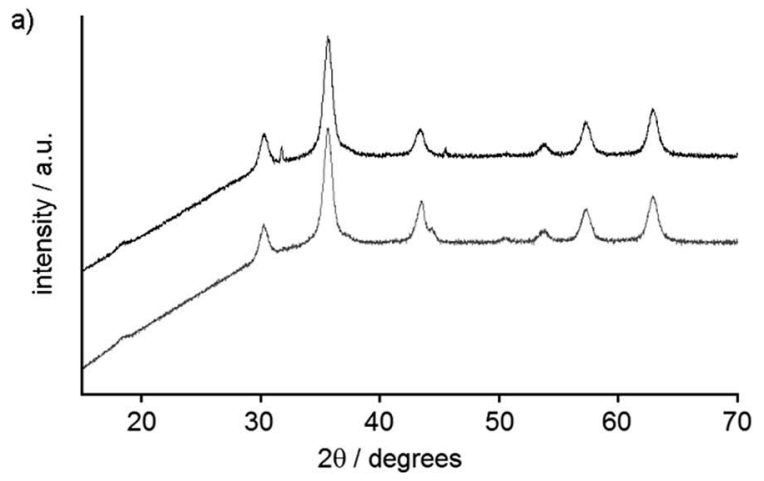

b)

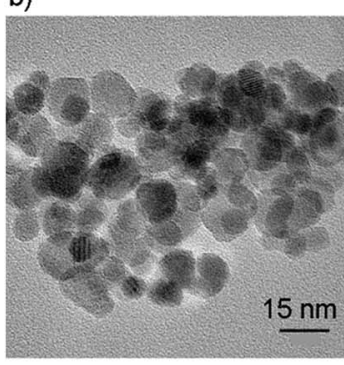

c)

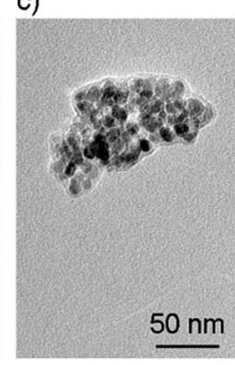

d)

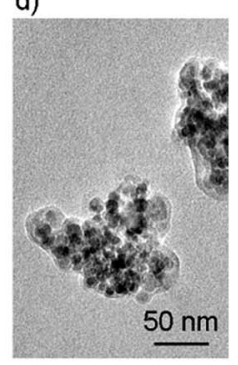

e)

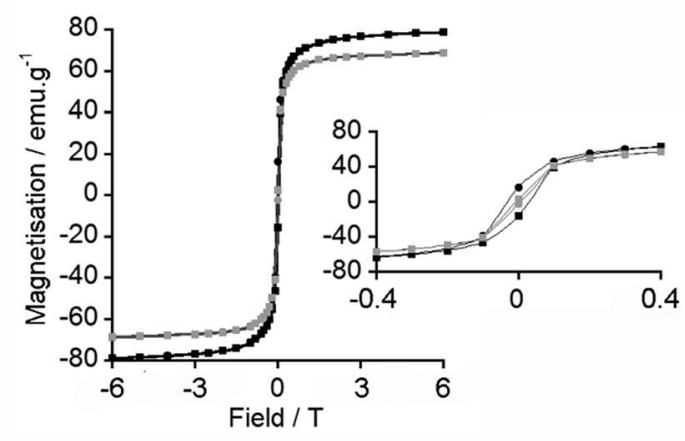

f)

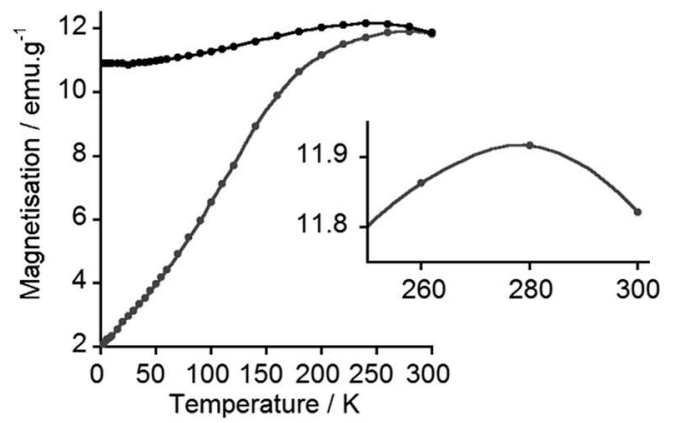

Fig. 3 (a) XRD spectra of uncoated MNPs (black) and GlcNAc-coated MNPs (gray, offset). (b) TEM image of uncoated MNPs. (c) TEM image of GlcNAc-coated MNPs showing cluster size and carbonaceous coating corona. (d) TEM image of GlcNAc-coated MNPs with WGA $(0.1 \mathrm{mg}$ $\mathrm{mL}^{-1}$ ). (e) SQUID measurement of magnetisation vs. field curves for uncoated MNPs at $5 \mathrm{~K}$ (black) and $300 \mathrm{~K}$ (gray). Inset: Expansion to show hysteresis. (f) SQUID measurement of ZFC (gray) and FC (black) curves for uncoated MNPs. Inset: Expanded section of ZFC curve showing blocking temperature of $278 \mathrm{~K}$.

wheat germ agglutinin (WGA), ${ }^{17}$ the addition of WGA to 5coated MNPs did not appear to significantly change the extent of interparticle aggregation (Fig. 3d). 
Dynamic light scattering (DLS) measurements were performed on uncoated MNPs and representative coated MNPs (coated with LacNAc conjugate $\mathbf{9}$, sialic acid conjugate $\mathbf{4}$ and the catechol hydrazide 1), which might be expected to provide coatings with neutral, anionic and cationic surface charges respectively. DLS indicated there was a significant amount of aggregation in Milli-Q water but the suspensions gave poor quality scattering data. Passing the suspensions through a 200 $\mu \mathrm{m}$ pore size filter resulted in better data, showing aggregates typically $80-200 \mathrm{~nm}$ in diameter ( $81 \mathrm{~nm}$ for uncoated and $153 \mathrm{~nm}$ for 4-coated MNPs), usually with larger populations also present ( $c a$. $>1000 \mathrm{~nm}$ diameter, see the ESI $\dagger$ ). In addition, changing the DLS scattering angle revealed particles in a freshly sonicated 9-coated MNP suspension that had a diameter of $7 \pm$ $2 \mathrm{~nm}$, close to that observed by TEM. The zeta potential was also determined for uncoated MNPs as well as MNPs coated with 1, 4 and 9. Uncoated particles were found to have a slightly anionic surface $(-15 \pm 8 \mathrm{mV})$, consistent with previous reports. ${ }^{38}$ Coating the particles with 9 produced little change in zeta potential compared to uncoated MNPs $(-13 \pm 8 \mathrm{mV})$, but the MNPs became more negative $(-35 \pm 4 \mathrm{mV})$ after coating with 4 and more positive $(-4 \pm 1 \mathrm{mV})$ after coating with 1 . The relatively low zeta potentials, which are reduced further in HEPES and PBS buffers (see ESI $\dagger$ ), may contribute to the propensity of these particles to aggregate. ${ }^{39}$

In order to determine if the particles were superparamagnetic iron oxide nanoparticles (SPIONs), the magnetic properties of uncoated MNPs were assessed using a Superconducting Quantum Interference Device (SQUID). Magnetisation $v s$. field curves revealed a coercive field of $0.31 \mathrm{kOe}$ at 5 $\mathrm{K}$, indicative of ferromagnetic behaviour, and a magnetic saturation of $79 \mathrm{emu}^{-1}$ at 6 Tesla (Fig. 3e). At $300 \mathrm{~K}$, no hysteresis was observed, confirming the nanoparticles as superparamagnetic at room temperature, with a magnetic saturation of $69 \mathrm{emu}^{-1}$ at 6 Tesla (Fig. 3e). As the primary use for these nanoparticles is envisioned to be in vivo biological imaging at ca. $310 \mathrm{~K}$, the SQUID data suggested these particles could be useful MRI contrast agents.

To determine the blocking temperature, zero-field cooled (ZFC) and field cooled (FC) measurements were performed under a 100 Oe field. The maximum point of the ZFC curve provides an estimate of the blocking temperature (Fig. 3f). ${ }^{40}$ This maximum at $278 \mathrm{~K}$ is higher than blocking temperatures commonly reported for dispersed $\mathrm{Fe}_{3} \mathrm{O}_{4}$ and $\gamma-\mathrm{Fe}_{2} \mathrm{O}_{3}$ MNPs, which are usually below $150 \mathrm{~K} .{ }^{41}$ Nonetheless higher temperatures have been reported, especially when particles have aggregated and there are significant interactions between the MNPs. ${ }^{42}$

\section{Magnetic resonance imaging (MRI) properties of GlcNAc- coated MNPs}

Given the SQUID data for uncoated MNPs, and to assess the effect of a saccharide coating on the ability of the MNPs to act as contrast agents, ${ }^{\mathbf{4 3}}$ the $T_{1}$ and $T_{2}$ relaxivity ${ }^{\mathbf{4}}$ of GlcNAc-coated MNPs was determined. These measurements were performed in an MRI machine equipped with a 9.4 T magnet using a multi- slice-multi-echo sequence. Five MNP suspensions with total iron concentrations, as measured by ICP-AES, up to $0.5 \mathrm{mM}$ in PBS (pH 7.4) were created. Agar ( $2 \mathrm{~g}$ ) was dissolved in PBS (100 $\mathrm{mL}, \mathrm{pH} 7.4$ ) and heated using a microwave oven until it was fully dissolved. GlcNAc-coated MNP suspensions $(1 \mathrm{~mL})$ were then mixed together with agar solution $(1 \mathrm{~mL})$. The agar decreases inhomogeneity due to MNP sedimentation and aggregation, and after cooling and thickening the gels contained an even dispersion of embedded nanoparticles. In addition, the lectin WGA (100 $\mu \mathrm{g}$ of $36 \mathrm{kDa}$ lectin, $5.6 \mathrm{nmol}$ binding sites) was mixed with 5-coated MNP suspensions and fixed in agar in the same way. Recognition of 5-coated MNPs by surface-bound WGA has been demonstrated using QCM-D ${ }^{17}$ but the effect of lectin binding on the magnetic properties of MNPs was unknown, for example a change in the extent of aggregation could alter $T_{2} \cdot{ }^{\mathbf{1 6}}$ Like many plant lectins, the lectin WGA has a relatively low affinity for its conjugate saccharides $\left(K_{\mathrm{d}}\right.$ for GlcNAc of $\left.760 \mu \mathrm{M}\right){ }^{45}$
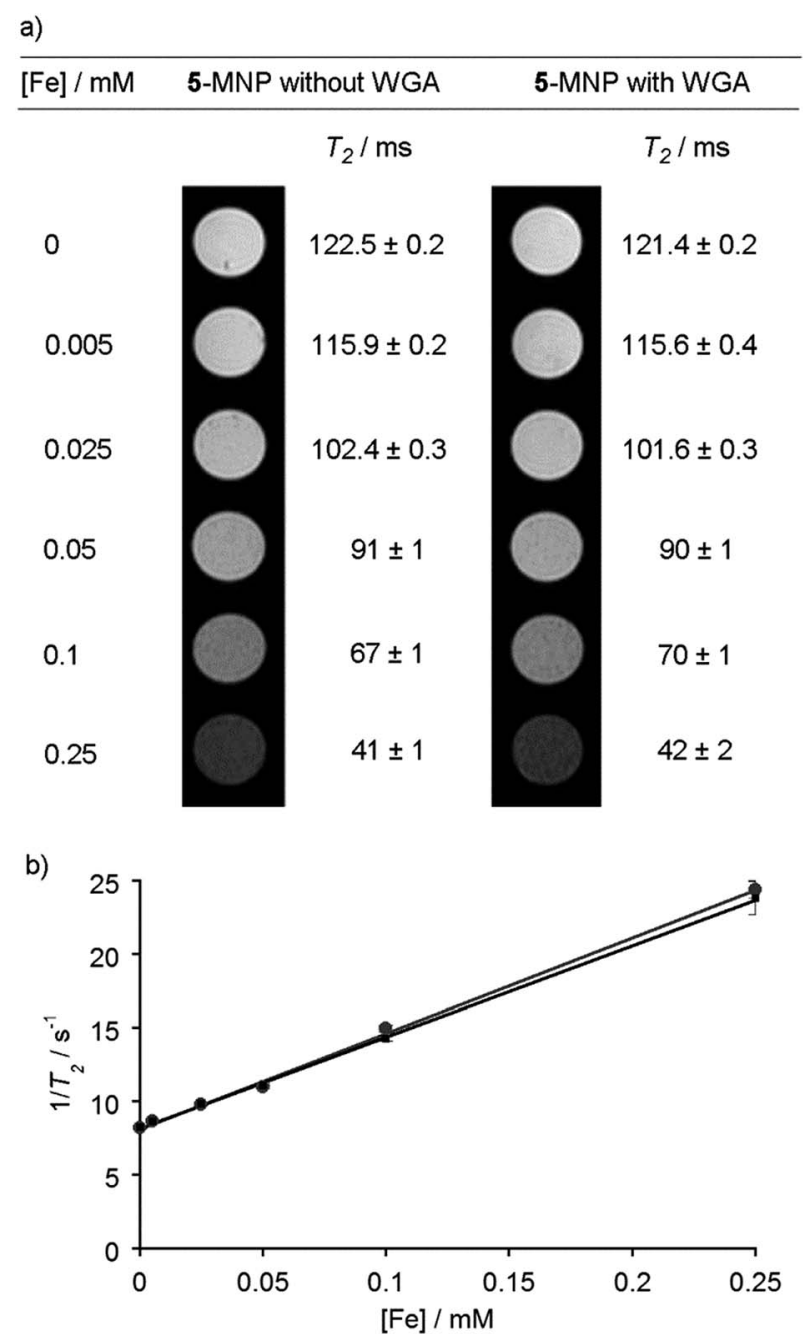

Fig. 4 (a) MRI contrast images of GlcNAc-coated MNPs in agar at different concentrations, both with and without wheat germ agglutinin (WGA). Measured $T_{2}$ values next to images. (b) Plot of iron concentration against $1 / T_{2}$ for GlcNAc-coated MNPs, with (black squares) and without WGA (grey circles). 
Although the concentration of GlcNAc on the MNPs (estimated as $13 \mu \mathrm{M}$ at $[\mathrm{Fe}]=0.25 \mathrm{mM}$, see the ESI $\dagger$ ) and WGA binding site concentration $(1.4 \mu \mathrm{M})$ are both lower than the $K_{\mathrm{d}}$, a cluster glycoside effect at the nanoparticle surface may strengthen recognition. ${ }^{46}$

As the concentration of GlcNAc-coated MNPs increased, the spin-spin relaxation time, $T_{2}$, decreased significantly, from 120 to $40 \mathrm{~ms}$, both in the presence and absence of WGA. This is displayed visually as a darkening of the image, from the bright white of pure agar in PBS, to the dark grey image obtained with $0.25 \mathrm{mM}$ [Fe] (Fig. 4a). The spin-lattice relaxation time, $T_{1}$, was relatively unchanged for both samples, slightly decreasing from 3300 to $2700 \mathrm{~ms}$ as the MNP concentration increased.

The $r_{2}$ value, which indicates the $T_{2}$ relaxivity of an MRI contrast agent, can be calculated from the gradient of a plot of total iron concentration against $1 / T_{2}$ (Fig. $4 \mathrm{~b}$ ). ${ }^{47}$ Without any WGA present, the $r_{2}$ value was calculated to be $(65 \pm 8) \mathrm{mM}^{-1} \mathrm{~s}^{-1}$, and in the presence of WGA the $r_{2}$ value was $(62 \pm 8) \mathrm{mM}^{-1} \mathrm{~s}^{-1}$. Both values compare well with other iron oxide MNPs of similar size formed by co-precipitation, and also with the values observed for previously-used clinical $T_{2}$ MRI agents such as Ferumoxtran $\left(60 \mathrm{mM}^{-1} \mathrm{~s}^{-1}\right){ }^{44,48,49}$ The corresponding $r_{1}$ values were low (both $(0.3 \pm 0.2) \mathrm{mM}^{-1} \mathrm{~s}^{-1}$, see the ESI $\left.\dagger\right)$ providing $r_{2} / r_{1}$ ratios in the order of 200, which suggests that this class of saccharide-coated MNP would give viable contrast agents. ${ }^{50}$ Taken together, these data indicate that surface reaction of the MNPs with the adduct 5 has not significantly diminished the desirable magnetic properties of the MNPs. However, the addition of lectin did not change $r_{2}$, which suggests that either insufficient lectin was bound to the MNP surface or the extent of MNP aggregation did not change sufficiently to produce a change in the relaxivity. The latter may be the case if the MNPs already have a tendency to agglomerate, as indicated by the DLS, TEM and SQUID data, whilst setting of the lectin/5-MNP mixtures in agar may have inhibited further aggregation.

\section{Conclusions}

The aniline-catalysed condensation of reducing saccharides with commercially available catechol-hydrazide $\mathbf{1}$ has been shown to be a versatile method for providing magnetic nanoparticles with saccharide coatings. A library of eleven different mono-, di- and trisaccharide adducts has been developed, although the condensation methodology was ineffective for the more complex and expensive saccharides that were tested (Lewis ${ }^{\mathrm{X}}$ trisaccharide and Lewis $^{\mathrm{X}}$ tetrasaccharide). The successful enzymatic transformation of GlcNAc-catechol adduct 5 into LacNAc-catechol adduct $\mathbf{9}$ suggests that chemoenzymatic methods could provide difficult-to-synthesise adducts, and give access to MNPs coated with complex oligosaccharides; synthetic GlcNAc derivatives have been elaborated into Lewis ${ }^{\mathrm{X}}$ and Lewis ${ }^{\mathrm{a}}$ through a series of enzymatic transformations. ${ }^{51}$ Although better HPLC methods for separating substrates from products should be developed, there is also the potential for these enzymatic transformations to be performed directly on adducts immobilised on the nanoparticle surface.
Iron oxide MNPs were synthesised by a co-precipitation method and shown to be $8 \mathrm{~nm}$ diameter SPIONs at room temperature $\left(T_{\mathrm{B}} \sim 278 \mathrm{~K}\right)$. The blocking temperature was higher than that commonly observed for dispersed iron oxide MNPs, and was consistent with MNP agglomeration. The observation of $200 \mathrm{~nm}$ diameter aggregates of uncoated MNPs and GlcNAccoated MNPs by TEM (in the presence and absence of the conjugate lectin) supported this proposition. TEM also showed that a GlcNAc coating did not produce significant morphological changes in individual MNPs. MRI measurements in agar showed that GlcNAc-coated particles had properties suitable as a MRI contrast agent, with shortening of the $T_{2}$ relaxation times with increasing MNP concentration $\left(r_{2}=(65 \pm 8) \mathrm{mM}^{-1} \mathrm{~s}^{-1}\right)$ but little change in the $T_{1}$ relaxation times. The addition of the conjugate lectin, WGA, did not significantly change these properties, perhaps because the size of the aggregates did not change significantly (as suggested by TEM data) or because the agar matrix inhibited further MNP aggregation. To provide saccharide coated MNPs that can detect lectins through aggregation-induced changes in magnetic properties, their intrinsic tendency to aggregate should be decreased, perhaps by increasing MNP surface charge (zeta potential) and/or the distance between the saccharide and the catechol surface anchor. Control over MNP aggregation will also be important for controlling MNP-cell interactions in vivo. ${ }^{52}$

The ability to rapidly obtain libraries of simple saccharide coatings using this high-throughput methodology should permit the creation of MNPs coated with mixtures of saccharides tailored to bind specific cell types. Preliminary data has shown that coating MNPs with 2 or 5 promotes recognition by 3T3 fibroblasts compared to uncoated MNPs, with indications that GlcNAc is better recognised than Glc by this cell line (although discrimination was relatively weak). ${ }^{17,18}$ Other cell lines may be more selective; we have shown HepG2 hepatocytes will take up LacNAc-coated lipid nanoparticles (liposomes) in preference to GlcNAc- and sialylLacNAc-coated liposomes, presumably through targeted binding to overexpressed asialoglycoprotein receptor on the surface of these liver cancer cells. ${ }^{26}$ Chemoenzymatic synthesis, as described here, of more complex coating adducts may also provide an additional level of cell selectivity. Selective recognition by non-adherent cells could provide applications in biomedical magnetophoresis, ${ }^{53}$ while in vivo MRI studies on animal models could confirm the performance of these MNPs as tissue contrast agents in a medically relevant context.

\section{Experimental}

General synthesis reagents and WGA were purchased from Sigma-Aldrich Co. Ltd. (Dorset, UK). 3,4-Dihydroxybenzhydrazide was supplied by Fluorochem (Derbyshire, UK) and $\mathrm{N}$ acetyllactosamine which was supplied by Carbosynth (Berkshire, UK). Permanent magnets were purchased from e-magnets UK, Hertfordshire, UK. Bovine $\beta$-1,4-galactosyltransferase 1 ( $\beta 4 \mathrm{Gal}-\mathrm{T} 1)$ was obtained as previously described. ${ }^{25}$

Reversed-phase HPLC purification was performed on an Agilent 1100 series system with an Agilent Eclipse XDB-C18 
(9.4 $\mathrm{mm} \times 250 \mathrm{~mm})$ column. NMR spectra were taken in deuterated solvents using a Bruker $400 \mathrm{MHz}$ Avance spectrometer with broadband probe or a Bruker $800 \mathrm{MHz}$ Avance III. NMR chemical shift values are referenced to residual peaks from non-deuterated solvent and measured in ppm. Multiples are reported as singlets $(\mathrm{s})$, doublets $(\mathrm{d})$, triplets $(\mathrm{t})$, multiplets (m) or a combination of the above and coupling constants are measured in Hertz. Electrospray mass spectrometry was performed on a Micromass LCT instrument using a Waters 2790 separations module with electrospray ionization and TOF fragment detection. High resolution mass spectrometry was performed on a Water Q-TOF micro with an ES+/- ion source. Elemental analysis was performed using a Thermo Scientific FLASH 2000 series CHNS/O Analyser. Sonication of nanoparticles (e.g. for coating) was performed with a Sonics VibraCell VCX 130PB Ultrasonic Processor (CV 188) with a stepped micro tip $(3 \mathrm{~mm} \times 136 \mathrm{~mm})$ running at $130 \mathrm{~W}, 20 \mathrm{kHz}$ and $50 \%$ amplitude. Bath sonication was carried out using a Camlab Transonic T460 operating at $35 \mathrm{kHz}$. Centrifugation was performed in $15 \mathrm{~mL}$ Falcon tubes using a Heraeus Megafuge 1.0R spinning at $4200 \mathrm{rpm}$ at a constant temperature of $23{ }^{\circ} \mathrm{C}$ for $10 \mathrm{~min}$.

\section{General procedure for the synthesis of adducts}

Saccharide $(0.3 \mathrm{mmol})$ and hydrazide $(0.3 \mathrm{mmol})$ were dissolved in methanol with aniline ( $10 \mathrm{~mL}$ of $5 \mathrm{mM}$ stock solution). The reaction was allowed to reflux overnight under a $\mathrm{N}_{2}$ atmosphere. After this time, the reaction was allowed to cool before removal of the solvent under reduced pressure. Purification was achieved by HPLC, with multiple aliquots $(0.5 \mathrm{~mL})$ of the reaction mixture separated on a semi-preparative column $(9.4 \mathrm{~mm} \times 250$ $\mathrm{mm}$ ) using a gradient method shifting linearly over $1 \mathrm{~h}$ (from $5 \%$ to $50 \%$ THF in water). The eluent was monitored by UVvisible spectroscopy (230 and/or $250 \mathrm{~nm}$ ), and the uncharged saccharide-catechol adducts typically eluted between 14 and $18 \mathrm{~min}$ ( $1 \mathrm{~mL} \mathrm{~min}^{-1}$ flow rate). The product containing fractions were collected and freeze-dried to give the adducts as white powders.

\section{Chemoenzymatic synthesis of 9}

Glycoconjugate 5 (10 $\mu \mathrm{mol})$ was dissolved in 2-( $N$-morpholino) ethanesulfonic acid (MES) buffer (1 mL, $50 \mathrm{mM}, \mathrm{pH} 7.0)$ and mixed with UDP-Gal (20 $\mu \mathrm{mol}, 11.25 \mathrm{mg})$ and $\beta 4 \mathrm{Gal}-\mathrm{T} 1$ (14.75 $\mu \mathrm{L}$ of a $0.54 \mathrm{mg} \mathrm{mL}^{-1}$ solution). After degassing the solution by sonication and purging it with argon, $\mathrm{MnCl}_{2}(3 \mu \mathrm{L}$ of a $1.0 \mathrm{M}$ solution in water) was added to the solution flask. The mixture was then vortex mixed followed by incubation overnight at $37{ }^{\circ} \mathrm{C}$. The reaction mixture was directly purified by HPLC (Macherey-Nagel Nucleosil C18, $250 \times 4.6 \mathrm{~mm}, 5 \mu \mathrm{m}) \mathrm{using}$ portion-wise $(20-80 \mu \mathrm{L})$ addition to an analytical column and a 40 min gradient ranging from $5 \%$ to $100 \%$ acetonitrile in

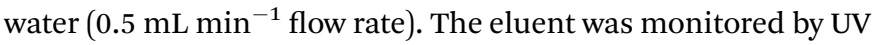
spectroscopy (260 nm) and the product containing fractions were collected and freeze-dried to afford a $7: 3$ mixture of compounds 5 and 9.

\section{General nanoparticle coating procedure}

Iron oxide nanoparticles were synthesised by adding $\mathrm{FeSO}_{4} \cdot 7 \mathrm{H}_{2} \mathrm{O}$ to $\mathrm{Fe}_{2}\left(\mathrm{SO}_{4}\right)_{3} \cdot \mathrm{H}_{2} \mathrm{O}$ in a $1: 2$ mole ratio in Milli-Q filtered water at $80{ }^{\circ} \mathrm{C}$ under a flow of $\mathrm{N}_{2}$. After vigorous stirring (15 min), $\mathrm{NH}_{4} \mathrm{OH}$ was added and stirred vigorously for a further $30 \mathrm{~min}$. The resultant MNPs were washed with Milli-Q filtered water until a neutral $\mathrm{pH}$ was obtained, re-suspended in a $\mathrm{NaCl}$ solution $(20 \mathrm{~mL}, 40 \mathrm{mM})$, lyophilised and stored under $\mathrm{N}_{2}$.

MNPs (10 mg) were suspended in methanol $(5 \mathrm{~mL})$ by probe sonication $(130 \mathrm{~W}, 20 \mathrm{kHz}, 50 \%$ amplitude) for 5 minutes. To this suspension was added the desired coating molecule (any of 2 to $12,10 \mathrm{mg}$ ). The sample was sonicated with a probe sonicator for a further $45 \mathrm{~min}$. Any unreacted coating material was removed by centrifugation $(4200 \mathrm{rpm}, 10 \mathrm{~min})$ to give a pellet then supernatant removal (pellet held in place with aid of a permanent ring magnet, $0.51 \mathrm{~T}$ ). The process of sedimentation and washing was repeated twice with methanol $(2 \times 10 \mathrm{~mL})$ and once with Milli-Q filtered water until the coated nanoparticles were finally suspended in Milli-Q filtered water $(3 \mathrm{~mL})$ and either used immediately or lyophilised for storage.

\section{X-ray power diffraction (XRD)}

XRD was performed at the University of Namur in the Namur Nanosafety Center/Namur Research Institute for Life Sciences. Diffraction patterns were obtained using a Philips PW3064 XPERT-PRO diffractometer using $\mathrm{Cu} \mathrm{K} \alpha$ radiation. The MNP powders were placed over the aperture on a slide.

\section{Transmission electron microscopy (TEM)}

TEM of uncoated nanoparticles was carried out using a Philips CM20 operated at $200 \mathrm{keV}$. Samples were prepared by suspending nanoparticles in methanol then dropping a dilute sample onto a carbon coated copper grid and leaving the solvent to evaporate. TEM of 5-MNPs in the absence and presence of lectin was carried out using a FEI Tecnai G2 20 operated at 200 $\mathrm{keV}$. Samples were prepared by suspending the nanoparticles in water $(1 \mathrm{~mL})$ at a concentration of $0.01 \mathrm{mg} \mathrm{mL}^{-1}$. Half of the volume of each sample was removed and incubated with WGA (0.1 $\mathrm{mg}, 5.6 \mathrm{nmol}$ binding sites) and incubated at room temperature for $4 \mathrm{~h}$. The other half of each suspension of MNPs was left untreated. The samples were dispersed by vigorous shaking, drops of each were placed on TEM grids, and the solvent left to evaporate.

\section{Dynamic light scattering (DLS) and zeta potential measurements}

DLS and zeta potential measurements (using the Smoluchowski equation) were carried out using a Malvern Zetasizer Nano ZSP $633 \mathrm{~nm}$ laser at $25^{\circ} \mathrm{C}$. Coated MNPs were suspended in PBS (pH 7.4), Milli-Q water or HEPES buffer (20 mM, pH 7.5 with $150 \mathrm{mM}$ $\mathrm{NaCl}$ and $2 \mathrm{mM} \mathrm{CaCl}_{2}$ ) at concentrations of $6 \times 10^{-7} \mathrm{mg} \mathrm{mL}^{-1}$. Particles were suspended by sonication with a probe-type sonicator for $10 \mathrm{~min}$ followed by filtration using a Minisart ${ }^{\circledR}$ syringe filter of $200 \mu \mathrm{m}$. Measurements were carried out in folded capillary zeta cells with a scattering angle of $13^{\circ}$ or $173^{\circ}$. 


\section{SQUID measurements of uncoated MNPs}

Magnetic studies were carried out using a Quantum Design MPMS-XL SQUID magnetometer equipped with a 7 Tesla magnet. In order to prevent the samples from adopting an orientation in the applied magnetic field they were immobilised in an eicosane matrix and placed in gelatine capsules. Magnetic hysteresis data were recorded at 5 and $300 \mathrm{~K}$, by cycling the magnetic field between $(6 \mathrm{~T})$ and $(-6 \mathrm{~T})$ fields (sequence used: $(0) \rightarrow(6 \mathrm{~T}) \rightarrow(-6 \mathrm{~T}) \rightarrow(6 \mathrm{~T}))$. Zero field cooled (ZFC) and field cooled (FC) magnetisation data were collected under an applied static magnetic field of 100 Oe. For ZFC, the sample was initially cooled from 300 to $5 \mathrm{~K}$ at a rate of $10 \mathrm{~K} \mathrm{~min}^{-1}$ under zero-dc field: no data was collected at this step. After keeping the temperature stable at $5 \mathrm{~K}$ for $5 \mathrm{~min}$, a small 100 Oe magnetic field was applied and ZFC data as a function of temperature was collected under a warming regime at $10 \mathrm{~K} \mathrm{~min}^{-1}$. For FC data, a field of 100 Oe was maintained when cooling the sample from 300 to $5 \mathrm{~K}$ without measurement, and then data were collected under same field upon warming from 5 to $300 \mathrm{~K}$ at $10 \mathrm{~K} \mathrm{~min}^{-1}$.

\section{Magnetic resonance imaging (MRI)}

GlcNAc-coated MNPs were suspended in PBS (1 mL, pH 7.4, 10 $\mathrm{mM}$ ) with Fe concentrations of $0.01 \mathrm{mM}, 0.05 \mathrm{mM}, 0.1 \mathrm{mM}$, $0.2 \mathrm{mM}$ and $0.5 \mathrm{mM}$. For lectin binding, 5-MNPs $(0.5 \mathrm{mM}$ Fe concentration) were suspended in PBS (2 mL, pH 7.4, $10 \mathrm{mM}$ ), then WGA $(100 \mu \mathrm{g}, 5.56 \mathrm{nmol}$ binding sites, in $50 \mu \mathrm{L}$ Milli-Q filtered water) was added and the suspension incubated at room temperature for $3 \mathrm{~h}$. Some of the sample was then diluted to make lower Fe concentrations of $0.01 \mathrm{mM}, 0.05 \mathrm{mM}, 0.1 \mathrm{mM}$ and $0.2 \mathrm{mM}$. Control samples with and without lectin were also made without the addition of MNPs.

To prepare the samples for MRI, agar powder ( $2 \mathrm{~g}$ ) was dissolved in PBS (100 mL, pH 7.4, $10 \mathrm{mM}$ ) by heating in a microwave oven for periods of approximately $40 \mathrm{~s}$, with stirring in between, until fully dissolved. The MNP suspensions in PBS were added to an Eppendorf tube, and an equal amount of liquid agar solution added by pipette, followed by careful mixing by pipette (avoiding bubble formation). The tubes containing MNPs in agar were left to cool to room temperature, forming a solid gel containing dispersed MNPs.

MRI was performed using a Bruker BioSpec Avance III 94/20 Preclinical MRI. MR images were acquired at 9.4 T using a 2D multi-slice multi-echo sequence at $300 \mathrm{~K}$ for simultaneous $T_{1}$ and $T_{2}$ measurements. ${ }^{37}$ For MRI measurements, Eppendorf tubes containing MNPs in agar were inserted into a holder, and the holder inserted into the instrument.

\section{Conflicts of interest}

There are no conflicts to declare.

\section{Acknowledgements}

This work was supported by the North-West Nanoscience Doctoral Training Centre and the EPSRC (grants EP/K039547/1, EP/G03737X/1 and EP/N009134/1). We thank Mrs R. Sung for her guidance in developing HPLC protocols. We also thank Dr R. Spiess and the Mass Spectrometry Service in the School of Chemistry (University of Manchester) for mass spectrometry support. We thank Mr P. Lythgoe (University of Manchester) for assistance with ICP-AES measurements. This research used the facilities supported by Microscopy Australia at the Electron Microscope Unit (at UNSW), Mark Wainwright Analytical Centre (MWAC, at UNSW) and the EPSRC UK National EPR Facility (at the University of Manchester). The assistance of Dr M. L. H. Gruwel (Biological Resources Imaging Laboratory, MWAC) with MRI measurements is gratefully acknowledged. We thank Dr J. Mejia and Ms O. Fichera at the University of Namur for assisting with XRD experiments, and we thank the Manchester Enterprise Centre and UMIP for an Innovation Optimiser Project Award that funded those experiments and the purchase of Lewis $\mathrm{X}$ saccharides.

\section{References}

1 Z. R. Stephen, F. M. Kievit and M. Zhang, Mater. Today, 2011, 14, 330-338.

2 D. Chang, M. Lim, J. A. C. M. Goos, R. Qiao, Y. Y. Ng, F. M. Mansfeld, M. Jackson, T. P. Davis and M. Kavallaris, Front. Pharmacol., 2018, 9, 831.

3 D. Fourmy, J. Carrey and V. Gigoux, Nanomedicine, 2015, 10, 893-896.

4 N. Pamme, J. C. T. Eijkel and A. Manz, J. Magn. Magn. Mater., 2006, 307, 237-244.

5 C. Carr, M. Espy, P. Nath, S. L. Martin, M. D. Ward and J. Martin, J. Magn. Magn. Mater., 2009, 321, 1440-1445.

6 Z. Cheng, A. Al Zaki, J. Z. Hui, V. R. Muzykantov and A. Tsourkas, Science, 2012, 338, 903-910.

7 M. E. Griffin and L. C. Hsieh-Wilson, Cell Chem. Biol., 2016, 23, 108-121.

8 (a) S. Sampaolesi, F. Nicotra and L. Russo, Future Med. Chem., 2019, 11, 43-60; (b) T. A. Swift, M. Duchi, S. A. Hill, D. Benito-Alifonso, R. L. Harniman, S. Sheikh, S. A. Davis, A. M. Seddon, H. M. Whitney, M. C. Galan and T. A. A. Oliver, Nanoscale, 2018, 10, 13908-13912; (c) S. Park, G.-H. Kim, S.-H. Park, J. Pai, D. Rathwell, J.-Y. Park, Y.-S. Kang and I. Shin, J. Am. Chem. Soc., 2015, 137(18), 5961-5968.

9 (a) A. D. A. Zottis, J. M. Beltrame, L. R. S. Lara, T. G. Costa, M. J. Feldhaus, R. Curi Pedrosa, F. Ourique, C. E. M. de Campos, E. de A. Isoppo, F. da Silva Miranda and B. Szpoganicz, Chem. Commun., 2015, 51, 11194-11197; (b) X. Yang, H. Hong, J. J. Grailer, I. J. Rowland, A. Javadi, S. A. Hurley, Y. Xiao, Y. Yang, Y. Zhang, R. J. Nickles, W. Cai, D. A. Steeber and S. Gong, Biomaterials, 2011, 32, 4151-4160; (c) N. Schleich, P. Sibret, P. Danhier, B. Ucakar, S. Laurent, R. N. Muller, C. Jerome, B. Gallez, V. Preat and F. Danhier, Int. J. Pharm., 2013, 447, 94-101; (d) J. Tuček, Z. Sofer, D. Bouša, M. Pumera, K. Holá, A. Malá, K. Poláková, M. Havrdová, K. Čépe, O. Tomanec and R. Zbořil, Nat. Commun., 2016, 7, 12879-12890; (e) Y. Wang and H. Gu, Adv. Mater., 2015, 27, 576-585; (f) S. Cheong, P. Ferguson, K. W. Feindel, I. F. Hermans, P. T. Callaghan, 
C. Meyer, A. Slocombe, C.-H. Su, F.-Y. Cheng, C.-S. Yeh, B. Ingham, M. F. Toney and R. D. Tilley, Angew. Chem., Int. Ed., 2011, 50, 4206-4209.

10 L. A. Bass, M. Wang, M. J. Welch and C. J. Anderson, Bioconjugate Chem., 2000, 11, 527-532.

11 (a) R. Misri, D. Meier, A. C. Yung, P. Kozlowski and U. O. Hafeli, Nanomedicine, 2012, 8, 1007-1016; (b) P. Beard, Interface Focus, 2011, 1, 602-631.

12 M. Rogosnitzky and S. Branch, BioMetals, 2016, 29, 365-376.

13 M. Gueron, J. Magn. Reson., 1975, 19, 58-66.

14 R. Hachani, M. Lowdell, M. Birchall, A. Hervault, D. Mertz, S. Begin-Coline and N. T. K. Thanh, Nanoscale, 2016, 8, 3278-3287.

15 Q. A. Pankhurst, J. Connolly, S. K. Jones and J. Dobson, J. Phys. D: Appl. Phys., 2003, 36, R167-R181.

16 J. S. Basuki, L. Esser, H. T. T. Duong, Q. Zhang, P. Wilson, M. R. Whittaker, D. M. Haddleton, C. Boyer and T. P. Davis, Chem. Sci., 2014, 5, 715-726.

17 T. P. Coxon, T. W. Fallows, J. E. Gough and S. J. Webb, Org. Biomol. Chem., 2015, 13, 10751-10761.

18 T. W. Fallows, T. P. Coxon, J. E. Gough and S. J. Webb, MRS Adv., 2017, 2, 1279-1284.

19 M. Wendeler, L. Grinberg, X. Wang, P. E. Dawson and M. Baca, Bioconjugate Chem., 2014, 25, 93-101.

20 P. Crisalli and E. T. Kool, J. Org. Chem., 2013, 78, 1184-1189. 21 O. R. Baudendistel, D. E. Wieland, M. S. Schmidt and V. Wittmann, Chem.-Eur. J., 2016, 22(48), 17359-17365.

22 C. J. Gray, M. J. Weissenborn, C. E. Eyers and S. L. Flitsch, Chem. Soc. Rev., 2013, 42, 6378-6405.

23 (a) R. Šardzík, A. P. Green, N. Laurent, P. Both, C. Fontana, J. Voglmeir, M. J. Weissenborn, R. Haddoub, P. Grassi, S. M. Haslam, G. Widmalm and S. L. Flitsch, J. Am. Chem. Soc., 2012, 134, 4521-4524; (b) N. Laurent, R. Haddoub, J. Voglmeir, S. C. C. Wong, S. J. Gaskell and S. L. Flitsch, ChemBioChem, 2008, 9, 2592-2596; (c) L. Ban and M. Mrksich, Angew. Chem., Int. Ed., 2008, 47, 3396-3399; (d) A. Sanchez-Ruiz, S. Serna, N. Ruiz, M. Martin-Lomas and N. C. Reichardt, Angew. Chem., Int. Ed., 2011, 50, 1801-1804.

24 (a) S. Serna, J. Etxebarria, N. Ruiz, M. Martin-Lomas and N. C. Reichardt, Chem.-Eur. J., 2010, 16, 13163-13175; (b) S. Park and I. Shin, Org. Lett., 2007, 9, 1675-1678.

25 G. T. Noble, F. L. Craven, J. Voglmeir, R. Šardzík, S. L. Flitsch and S. J. Webb, J. Am. Chem. Soc., 2012, 134, 13010-13017.

26 F. L. Craven, J. Silva, M. D. Segarra-Maset, K. Huang, P. Both, J. E. Gough, S. L. Flitsch and S. J. Webb, Chem. Commun., 2018, 54, 1347-1350.

27 (a) A. J. McGrath, S. Cheong, A. M. Henning, J. J. Gooding and R. D. Tilley, Chem. Commun., 2017, 53, 11548-11551; (b) J. H. L. Beal, S. Prabakar, N. Gaston, G. B. Teh, P. G. Etchegoin, G. Williams and R. D. Tilley, Chem. Mater., 2011, 23, 2514-2517; (c) N. A. Frey, S. Peng, K. Cheng and S. Sun, Chem. Soc. Rev., 2009, 38, 2532-2542; (d) R. Hao, R. Xing, Z. Xu, Y. Hou, S. Gao and S. Sun, Adv. Mater., 2010, 22, 2729-2742.
28 A. E. Rawlings, J. P. Bramble, R. Walker, J. Bain, J. M. Galloway and S. S. Staniland, Proc. Natl. Acad. Sci. U. S. A., 2014, 111, 16094-16099.

29 Y. S. Kang, S. Risbud, J. F. Rabolt and P. Stroeve, Chem. Mater., 1996, 8, 2209-2211.

30 P. Majewski and B. Thierry, Crit. Rev. Solid State Mater. Sci., 2007, 32, 203-215.

31 J. Baumgartner, L. Bertinetti, M. Widdrat, A. M. Hirt and D. Faivre, PLoS One, 2013, 8, e57070.

32 S. Nazrazadani and A. Raman, Corros. Sci., 1993, 34, 13551365.

33 W. Kim, C.-Y. Suh, S.-W. Cho, K.-M. Roh, H. Kwon, K. Song and I.-J. Shon, Talanta, 2012, 94, 348-352.

34 (a) M. Aghazadeh, I. Karimzadeh and M. R. Ganjali, J. Magn. Magn. Mater., 2017, 439, 312-319; (b) X. Zhu, J. Fan, Y. Zhang, H. Zhu, B. Dai, M. Yan and Y. Ren, J. Mater. Sci., 2017, 52, 12717-12723.

35 F. de Cogan, A. Booth, J. E. Gough and S. J. Webb, Angew. Chem., Int. Ed., 2011, 50, 12290-12293.

36 (a) F.-Y. Cheng, C.-H. Su, Y.-S. Yang, C.-S. Yeh, C.-Y. Tsai, C.-L. Wu, M.-T. Wu and D.-B. Shieh, Biomaterials, 2005, 26, 729-738; (b) P. I. P. Soares, A. M. R. Alves, L. C. J. Pereira, J. T. Coutinho, I. M. M. Ferreira, C. M. M. Novo and J. P. M. R. Borges, J. Colloid Interface Sci., 2014, 419, 46-51.

37 A. J. McGrath, C. Dolan, S. Cheong, D. A. J. Herman, B. Naysmith, F. Zong, P. Galvosas, K. J. Farrand, I. F. Hermans, M. Brimble, D. E. Williams, J. Jin and R. D. Tilley, J. Magn. Magn. Mater., 2017, 439, 251-258.

38 D. D. Jurašin, M. Curlin, I. Capjak, T. Crnković, M. Lovrić, M. Babič, D. Horák, I. V. Vrček and S. Gajović, Beilstein J. Nanotechnol., 2016, 7, 246-262.

39 S. Bhattacharjee, J. Controlled Release, 2016, 235, 337-351.

40 (a) I. J. Bruvera, P. Mendoza Zélis, M. Pilar Calatayud, G. F. Goya and F. H. Sánchez, J. Appl. Phys., 2015, 118, 184304; (b) C. Schmitz-Antoniak, Rep. Prog. Phys., 2015, 78, 062501.

41 T. Ozkaya, M. S. Toprak, A. Baykal, H. Kavas, Y. Köseoğlu and B. Aktaş, J. Alloys Compd., 2009, 472, 18-23.

42 (a) Y. A. Barnakov, M. H. Yu and Z. Rosenzweig, Langmuir, 2005, 21, 7524-7527; (b) S. Fleutot, G. L. Nealon, M. Pauly, B. P. Pichon, C. Leuvrey, M. Drillon, J.-L. Gallani, D. Guillon, B. Donnio and S. Begin-Colin, Nanoscale, 2013, 5, 1507-1516; (c) D. K. Kim, Y. Zhang, W. Voit, K. V. Rao and M. Muhammed, J. Magn. Magn. Mater., 2001, 225, 30-36.

43 M. F. Casula, A. Corrias, P. Arosio, A. Lascialfari, T. Sen, P. Floris and I. J. Bruce, J. Colloid Interface Sci., 2011, 357, 50-55.

44 N. Lee and T. Hyeon, Chem. Soc. Rev., 2012, 41, 2575-2589. 45 Y. Nagata and M. M. Burger, J. Biol. Chem., 1974, 249, 31163122.

46 (a) G. T. Noble, S. L. Flitsch, K. P. Liem and S. J. Webb, Org. Biomol. Chem., 2009, 7, 5245-5254; (b) J. J. Lundquist and E. J. Toone, Chem. Rev., 2002, 102, 555-578; (c) S. Won, S.-J. Richards, M. Walker and M. I. Gibson, Nanoscale Horiz., 2017, 2, 106-109; (d) S. A. Hill, D. Benito-Alifonso, D. J. Morgan, S. A. Davis, M. Berry and M. C. Galan, Nanoscale, 2016, 8, 18630-18634. 
47 N. Lee, H. Kim, S. H. Choi, M. Park, D. Kim, H.-C. Kim, Y. Choi, S. Lin, B. H. Kim, H. S. Jung, H. Kim, K. S. Park, W. K. Moon and T. Hyeon, Proc. Natl. Acad. Sci. U. S. A., 2011, 108, 2662-2667.

48 Y.-X. J. Wang, Quant. Imaging Med. Surg., 2011, 1, 35-40.

49 F. Hu, K. W. MacRenaris, E. A. Waters, T. Liang, E. A. Schultz-Sikma, A. L. Eckermann and T. J. Meade, J. Phys. Chem. C, 2009, 113, 20855-20860.

50 R. Hachani, M. A. Birchall, M. W. Lowdell, G. Kasparis, L. D. Tung, B. B. Manshian, S. J. Soenen, W. Gsell, U. Himmelreich, C. A. Gharagouzloo, S. Sridhar and N. T. K. Thanh, Sci. Rep., 2017, 7, 7850.
51 (a) I. Sittel and M. C. Galan, Org. Biomol. Chem., 2017, 15, 3575-3579; (b) M. C. Galan, A. T. Tran, K. Bromfield, S. Rabbani and B. Ernst, Org. Biomol. Chem., 2012, 10, 7091-7097.

52 H. H. Gustafson, D. Holt-Casper, D. W. Grainger and H. Ghandehari, Nano Today, 2015, 10, 487-510.

53 J. H. Kang, M. Super, C. W. Yung, R. M. Cooper, K. Domansky, A. R. Graveline, T. Mammoto, J. B. Berthet, H. Tobin, M. J. Cartwright, A. L. Watters, M. Rottman, A. Waterhouse, A. Mammoto, N. Gamini, M. J. Rodas, A. Kole, A. Jiang, T. M. Valentin, A. Diaz, K. Takahashi and D. E. Ingber, Nat. Med., 2014, 20, 1211-1216. 